\title{
Using multimedia modules to better prepare students for introductory physics lecture
}

\author{
Zhongzhou Chen, Timothy Stelzer, and Gary Gladding \\ Department of Physics, University of Illinois at Urbana-Champaign, Urbana, Illinois 61801, USA
}

(Received 26 January 2010; published 11 June 2010)

\begin{abstract}
It is known that introductory physics students rarely, if ever, read the textbook prior to coming to lecture. In this study, we report results from a curriculum intervention in a large enrollment introductory physics class that addresses this problem. In particular, we introduced web-based multimedia learning modules (MLMs) as a "prelecture assignment" designed to better prepare students before coming to lecture. We used student performance on "preflight questions" that they answer prior to lecture as a measure of their before-lecture understanding of the physics concepts. We found significant improvement in student performance and on the vast majority of these preflight questions as compared to that from previous semesters in which MLMs were not available. We found significant improvement for all students, independent of their background or ability level.
\end{abstract}

DOI: 10.1103/PhysRevSTPER.6.010108

PACS number(s): 01.40.Fk, 01.50.ht

\section{INTRODUCTION}

In a recent paper, [1] we reported the results of a clinical study in which we demonstrated that students receiving multimedia instruction significantly outperformed students who received equivalent textbook instruction, on both immediate postlesson assessments and a retention test given two weeks later. We observed effect sizes of 0.7 for both tests, consistent with those from the multimedia learning literature. [2]

We report here the first results from a systematic implementation of these multimedia learning modules (MLMs) in a large enrollment introductory physics course. We have chosen to use these modules as "prelectures," a web-based assignment that students are required to complete prior to attending lecture. Our motivation for this choice is simply to provide students with an initial exposure to the content before coming to lecture. It has been our experience, [1] as well as others [3-5] that asking students to read the textbook prior to coming to lecture is not an effective strategy to provide such an initial exposure.

The use of MLMs to provide this initial exposure to content, has two distinct advantages. First, MLMs are more effective than traditional textbooks for learning introductory physics content. [1] Second, since MLMs are web-based activities, student participation can be documented and awarded credit, thereby increasing compliance. In this paper we will demonstrate that students' conceptual understanding of certain basic physics concepts prior to coming to lecture are improved by the use of MLMs.

\section{IMPLEMENTATION OF MLMS AND JITT}

We created a set of 27 web-based multimedia learning modules for use in the introductory calculus-based electricity and magnetism course at the University of Illinois during the spring semester of 2008. Each prelecture is divided into approximately ten scenes, with each scene implemented as a Flash [8] movie containing dynamic animations synchronized with an audio narration that could be controlled by the student (pause, play, rewind, and position). Embedded formative assessments were included in two or three of the scenes for each module. These assessments are constructed as multiple-choice questions that must be answered correctly before the student can proceed to the next scene. The prelectures relied on multimedia learning principles to minimize cognitive load and improve students' learning of basic concepts, terminologies, as well as simple example calculations. The total narration time for a typical prelecture was about 15 min, during which we introduced most of the contents that would be covered in the upcoming lecture. The current versions of the MLMs for this course are available from our website [9].

During the semester, we logged all student interactions with the MLMs. In particular, we recorded the date and time whenever a student opened a prelecture, moved between scenes, and left a prelecture. Consequently, we can determine the time spent by each student viewing each scene of the prelecture. Students earned 2 credit points (out of 1000 total credit points for the course) by completing each prelecture.

Once a student completes the prelecture, they are presented with another assignment (called a "preflight") that they are asked to complete prior to lecture. This web-based assignment consists of a set of JiTT questions. These questions are generally multiple-choice conceptual questions concerning the main topics presented in the prelecture. Students are also asked to provide a free-response explanation for their choice of answer for each question. Each preflight assignment is worth 1 credit point, with full credit being given if the student responds in good faith, independent of the correctness of their answers. (Two examples of preflight questions are shown in Figs. 4 and 5.)

We then used the student performance on these preflight questions to design the lectures which included extensive peer instruction (generally eight to ten clicker questions per 50 min lecture). While MLMs were introduced into the course for the first time in Spring 2008, we had introduced preflights some years earlier. Consequently, we chose to repeat in Spring 2008 some prefight questions that we had given in previous years. We will use these repeated preflight questions to assess the effectiveness of the MLMs in preparing students for lecture.

\section{RESULTS AND DISCUSSION}

We chose to repeat in Spring 2008 a total of 64 preflight questions that had been used previously in both Spring 2006 


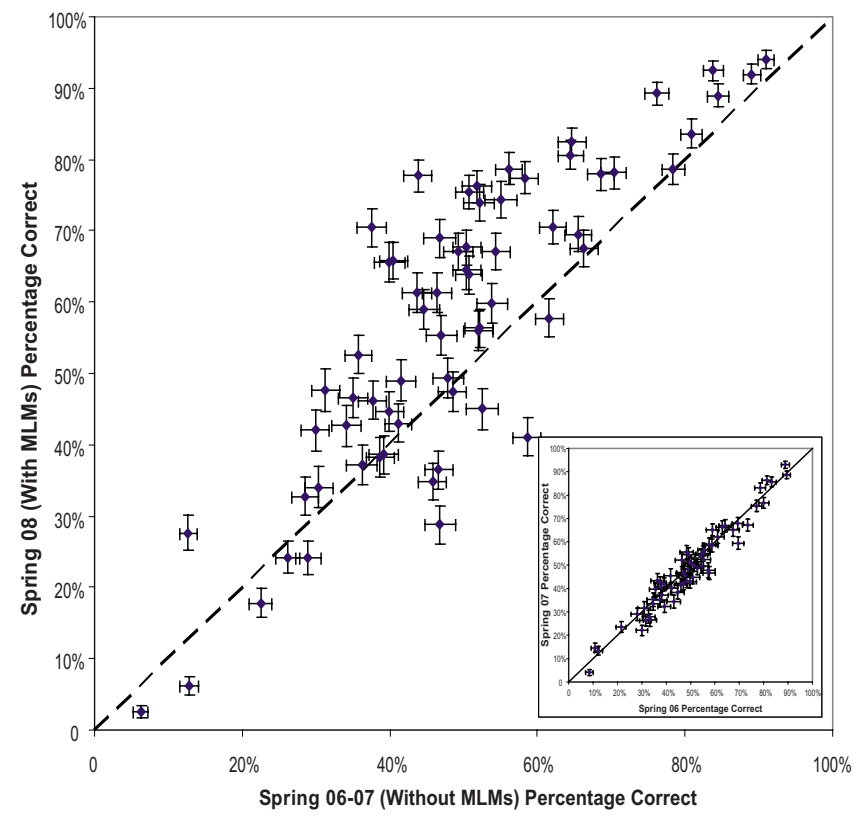

FIG. 1. (Color) Scatter plot of students' performance on 64 selected preflight questions. Each point represents one preflight question. The $x$ coordinate of a dot represents the average percentage of correct answers for that problem among all the students in Spring 06 and Spring 07 semesters (without prelecture intervention). The $y$ coordinate is the average percentage of correct answer for the same problem from students in Spring 08 semester (with prelecture intervention). The error bars represent the statistical uncertainty for each question assuming a binomial distribution. For the same set of preflight questions, we plot in the inset performance data from students in Spring 06 semester vs. students in Spring 07 semester. Here, all points clustered around the $y=x$ line, in sharp contrast with the asymmetric distribution in the main graph.

and Spring 2007. The questions covered content from 16 of the total of 27 lectures. A comparison of student performance shows that prelectures had significant impact on students' understanding of basic physics knowledge upon coming into lecture.

Shown in the inset of Fig. 1 is a scatter plot of the average scores on each of these 64 questions for students enrolled in two semesters (Spring 2006 and Spring 2007) prior to the introduction of MLMs. All data points cluster around the $y$ $=x$ line, showing that the average score on each question in Spring 2006 is statistically equivalent to its average score in Spring 2007 (the mean score of 64 preflight questions in Spring 2006 is $49.6 \pm 0.7 \%$ and the mean score in Spring 2007 is $48.6 \pm 0.7 \%$ ). One quantitative measure of the tightness of this cluster is that there is no question for which the difference in the average scores in Spring 2006 and Spring 2007 is different from zero by more than 3 standard errors $(3 \sigma)$, in agreement with the statistical expectation. Further, this average score is well-measured since the average enrollment in this course in these semesters is about 400 students (423 students in Spring 2006 and 379 for Spring 2007), and on average, $75-80 \%$ of the students completed the preflight assignment. We therefore take the average of the Spring 2006 and Spring 2007 scores on each question to obtain our baseline, our prediction for the average scores on each of these questions for the Spring 2008 had they not been given the MLMs as prelectures. We take the average scores on the 64 repeated preflight questions as a measure of the "beforelecture" understanding of these topics for each student population.

Figure 1 shows the comparison of the average score on these 64 repeated preflight questions for Spring 2008 students (mean score $57.3 \pm 0.7 \%$ ) to that of the Spring06/07 students (mean score 49 $\pm 0.7 \%$ ). [10] A clear improvement in student performance is seen for the vast majority of questions. Indeed, the average scores for 29 of the questions in Spring 2008 have increased from their expected value by more than $3 \sigma$ [11].

We attribute the significantly improved preflight performance of the Spring 2008 students to their exposure to the MLMs. We can strengthen this interpretation by studying the logs of student use of these MLMs. In particular, we observe a subset of the Spring 2008 students who do earn credit for the prelecture without fully participating in the activity. Namely, students can choose to skip the narration or animation on any given scene, except those that contain questions. The fraction of students that exhibit this behavior increases as the semester proceeds, presumably due to increased demands on their time from other commitments. If the improvement in the preflight performance is indeed due to the MLMs, we expect that these students who choose to skip most of the scenes should not demonstrate the significant improvement on the preflight questions.

We define a student to be a "nonviewer" for a given prelecture if that student skips (i.e., spends less than $75 \%$ of the audio narration time) more than two scenes. Typically, $30-40 \%$ of the students are classified as "nonviewers" for a given prelecture. Note that this definition is made on a prelecture by prelecture basis. A student can be a "viewer" for one prelecture and a "nonviewer" for a different prelecture.

Shown in Fig. 2 is a scatterplot of the performance on the repeated preflight questions in Spring08 vs. the performance in Spring06/07 for only those students who were defined as "viewers." The same plot for those students classified as "nonviewers" is shown in the inset. The difference in these scatterplots is striking. The performance of the "nonviewers" is quite similar to that of the Spring06 and Spring07 students (mean score $49 \pm 1.2 \%$ ), while the performance of the "viewers" is significantly better than that of the Spring06 and Spring07 students (mean score $65 \pm 0.8 \%$ ). Indeed, for the nonviewers, three questions showed a significant increase in the average score (more than $3 \sigma$ different) compared to the Spring06 and Spring07 averages, while three questions showed a significant decrease in the average score (more than $3 \sigma$ different) compared to the Spring06 and Spring07 averages. For the viewers, 34 questions showed a significant increase in the average score (more than $3 \sigma$ different) compared to the Spring06 and Spring07 averages, while only two questions showed a significant decrease in the average score (more than $3 \sigma$ different) compared to the Spring06 and Spring07 averages.

We attribute this striking difference in performance to the difference in the quality of the interaction with the MLMs between the "viewers" and "nonviewers." Alternatively, one 


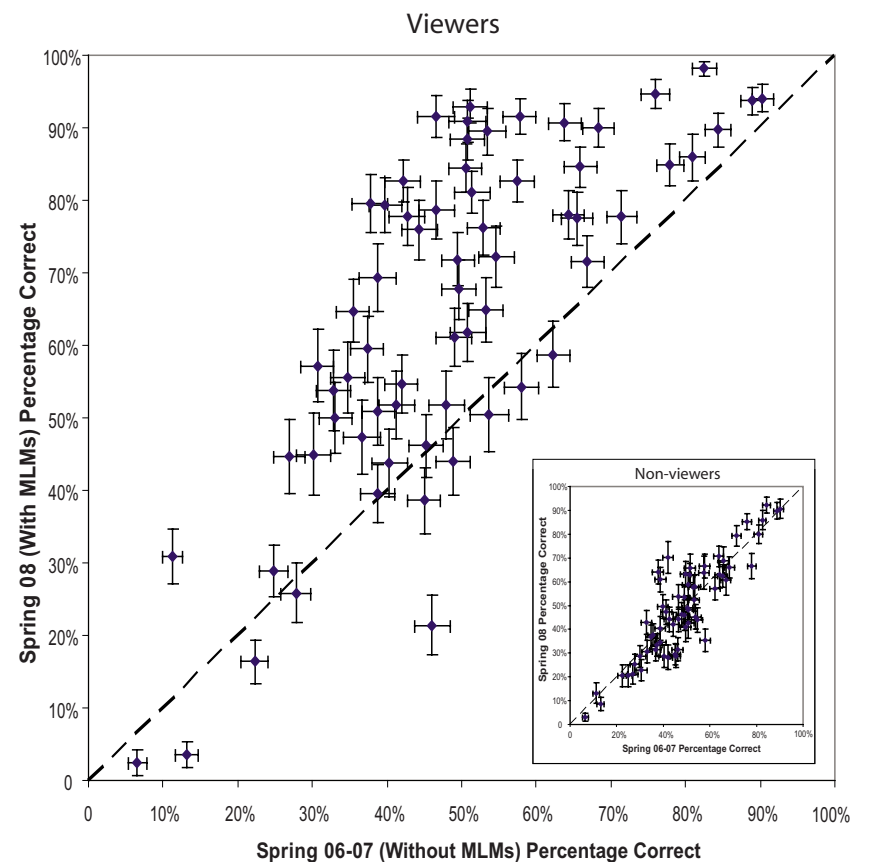

FIG. 2. (Color) Preflight performance of viewers in Spring 08 semester. The $y$-coordinate of each data point represents the percentage of correct answer for one preflight question among viewers in Spring 08 semester. The $x$-axis is the same as in Fig. 1. Inset: preflight performance of Spring 08 nonviewers.

might also argue that the performance difference could be due to intrinsic differences among these two populations, such as math skills, physics background, or attitude, since these populations are self-selected. We point to the symmetric distribution of the average scores about the $y=x$ line in the inset to Fig. 2 to argue against these alternative explanations. Namely, the "nonviewers" seem to be indistinguishable from all students in Spring06 and Spring07. If only the students who are either weaker or less motivated chose to be "nonviewers," we would expect the Spring06 and Spring07 students to outperform the "nonviewers," which is not the case. Therefore, we attribute the improved performance of the Spring08 students on the preflight questions directly to
TABLE I. Average preflight correct rate for different groups of students.

\begin{tabular}{lccc}
\hline \hline & A level & B Level & C Level \\
\hline Spring 08 viewer & $70.5 \pm 1.8 \%$ & $62.7 \pm 2.5 \%$ & $55.4 \pm 2.5 \%$ \\
Spring 08 nonviewer & $55.3 \pm 2.5 \%$ & $48.4 \pm 2.6 \%$ & $43.7 \pm 2.5 \%$ \\
Spring06/07 average & $54.9 \pm 1.3 \%$ & $45.1 \pm 1.1 \%$ & $45.1 \pm 1.2 \%$ \\
\hline \hline
\end{tabular}

the introduction of the MLMs into the course.

We will now strengthen this argument by explicitly grouping students based on their ability or background levels, and demonstrate that the observed improvement does not depend upon the ability or background of the students. In other words, we will show that MLMs do not preferentially benefit a certain class of students, but benefit uniformly across the broad spectrum of abilities and backgrounds of the students. To address this question, we define three populations of students, based on their performance in the preceding mechanics course. [12] Of the total of 1000 points available in the preceding mechanics course, we define Level A students as those students obtaining more than 885 points, Level B students as those students obtaining between 821 and 885 points, and $\mathrm{C}$ level students as those students obtaining between 690 and 820 points. [13]

We repeat the previous preflight analysis for each population of students. In Fig. 3, we show scatterplots of the average scores on the 64 questions in Spring08 vs the average scores on these questions in Spring06 and Spring07. The main plot is made for "viewers" and the inset plot is made for "nonviewers." Here we see that for all populations, the performance of the "nonviewers" is similar, on average, to that of the Spring06 and Spring07 students (the difference in mean scores stay within statistical fluctuation), while the performance of the "viewers" is significantly better than that of the Spring06 and Spring07 students. Table I shows the average scores on the set of 64 repeated questions for each student population (Levels A, B, and C) for Spring 06/07, Spring08 "nonviewers" and Spring08 "viewers."

There are two main results we take from these data. First, significant improvements are made by the "viewers" in all
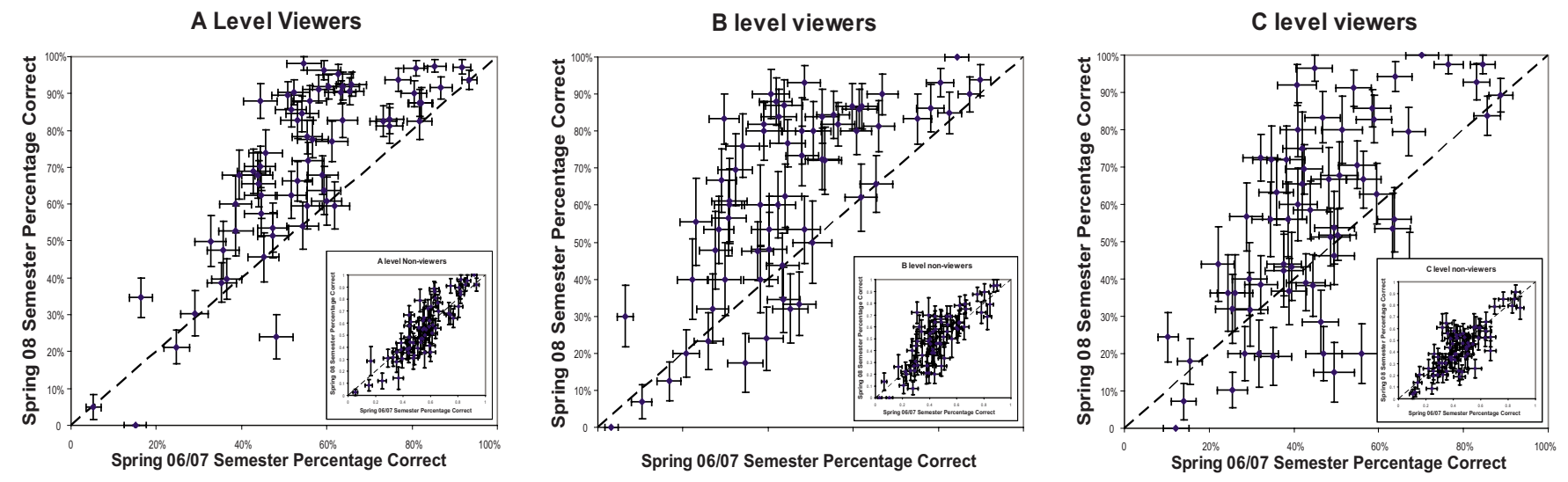

FIG. 3. (Color) Preflight performance of students with different levels of background knowledge. Shown in graphs A, B, and C are the preflight performance data of A, B, and C level viewers, respectively. The $x$ and $y$ axis have the same meaning as in Figs. 1 and 2, while the $x$ and $y$ values of each point is obtained from students within each level. The same data for nonviewers are plotted in the insets. 
1) The three configurations shown below are constuucted using identical capacitor

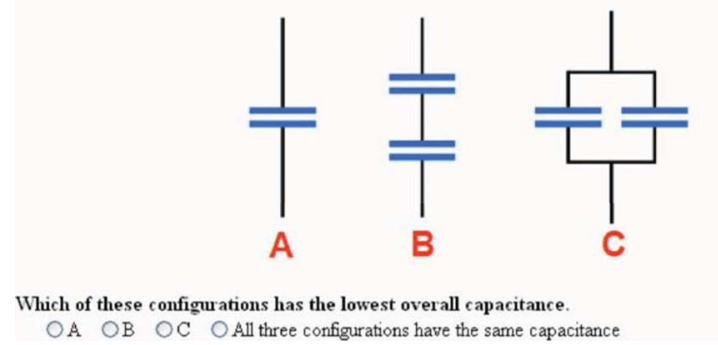

FIG. 4. (Color) Example of a preflight question.

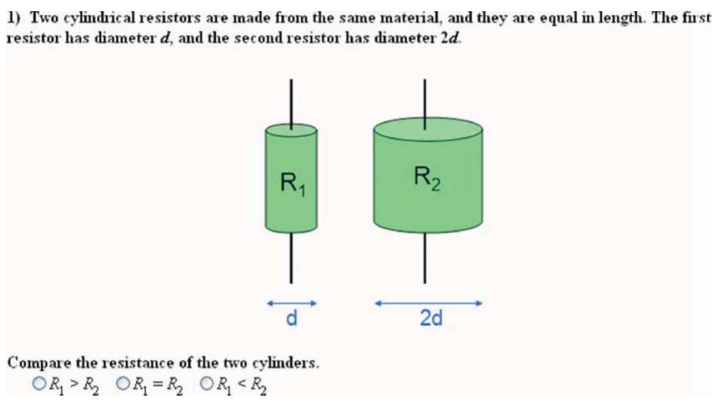

FIG. 5. (Color) Example of a preflight question.

provement on the vast majority of these questions; this improvement was observed for all students, independent of their background or ability level. Further, we observed that a subset of the students chose to skip the narration or animations in the prelectures and that the performance of these students was largely consistent with that of students from previous semesters in which the prelectures were not available.

In another paper [6], we will show that this improved understanding better prepares students for a more informed and interactive lecture experience. We observed significant improvements in performance on the vast majority of questions, however, we also notice that the actual amount of improvement is not uniform and varies considerably from question to question. The variance in improvement shows is not correlated with the difference in difficulty level of the problems. This suggests that the current implementation of multimedia technique is more effective for teaching some physics concepts than others. One possible explanation of the variance is that the questions showing the most improvement are the ones that cover topics whose understanding relies heavily on visualization of abstract concepts. The use of animation might help to release the heavy cognitive load that is incurred on the short term visual memory of the students [7]. Understanding where and why physics instruction can be improved through the use of multimedia will be an important contribution to future development of instructional materials.
[1] T. Stelzer, G. Gladding, J. Mestre, and D. T. Brooks, Comparing the efficacy of multimedia modules with traditional textbooks for learning introductory physics content. Am. J. Phys. 77, 184 (2009).

[2] R. E. Mayer, Multimedia Learning (Cambridge University Press, Cambridge, England, 2001).

[3] K. Cummings, T. French, and P. J. Cooney, Student textbook use in introductory physics, in Proceedings of the 2002 Physics Education Research Conference, edited by F. S. J. Marx and K. Cummings (PERC, New York, 2002).

[4] B. D. Smith and D. C. Jacobs, TextRev: A window into how general and organic chemistry students use textbook resources. J. Chem. Educ. Res. 80, 99 (2003).
[5] N. S. Podolefsky and N. D. Finkelstein, The perceived value of college physics textbooks: Students and instructors may not see eye to eye. Phys. Teach. 44, 338 (2006).

[6] T. Stelzer, D. T. Brookes, G. Gladding, and J. P. Mestre, Comparing the efficacy of multimedia modules with traditional textbooks for learning introductory physics content. Am. J. Phys. (to be published).

[7] P. Chandler and J. Sweller, Cognitive load theory and the format of instruction. Cogn. Instruct. 8, 293 (1991).

[8] http://en.wikipedia.org/wiki/Adobe_Flash

[9] To experience these prelectures as would a student, please visit: https://online-s.physics.uiuc.edu/courses/phys212/gtm/ No_Login/page.html 
[10] The average score of a question is the percentage of correct answer on the multiple-choice part of that question; students' free form response is not considered in the present study.

[11] We should note that the average scores on five questions decreased by more than $3 \sigma$, indicating another effect (smaller in magnitude) that can be attributed to the introduction of the MLMs. The naive interpretation that students experiencing the prelectures have less knowledge on these topics than their predecessors does not hold up to further investigation. These questions all had low average scores in Spring06/07, with the prevalent answer choice being either random or guided by intuition (e.g., if A doubles, then B doubles). For example, one question asked whether a person sanding on the edge of a pool of water could be prevented from seeing a light on the bottom of pool by total internal reflection at the water-air surface. In Spring06/07, 53\% answered "yes" and 47\% answered "no," while in Spring 2008, 72\% answered "yes" and 28\% answered "no." We interpret the increase in the percentage of incorrect answers in Spring 2008 due to partial knowledge (that light moving from water to air can be totally internally reflected) of the relevant physics that the Spring06/07 students did not have.
[12] Historically, about $65 \%$ of the $\mathrm{A}$ and $\mathrm{C}$ level students in the mechanics course remained $\mathrm{A}$ and $\mathrm{C}$ level students in electricity and magnetism course. For B level students, the corresponding fraction is about $40 \%$. Almost all changes are to adjacent levels; only a total of $5 \%$ of the students changed either from A level to $\mathrm{C}$ level or from $\mathrm{C}$ level to A level.

[13] The average number of A, B, and C level viewers in Spring 08 are:62, 29, 31; the average number of $\mathrm{A}, \mathrm{B}$, and $\mathrm{C}$ level nonviewers are:30, 24, 33; The average number of $\mathrm{A}, \mathrm{B}$ and $\mathrm{C}$ level students in Spring06/07 are:177, 151, 128.

[14] There is some evidence that the overall improvement by the $\mathrm{C}$ level students $(10-12 \%)$ is somewhat less than that of the A and B level students (14-17\%) and that this difference is mainly attributed to smaller improvements by the C level students on the most difficult questions. Namely, if we divide the questions into two sets based on difficulty as determined by the average score made by Spring06 and Spring07 students, we find that for the questions from the most difficult half, the improvement by $\mathrm{C}$ level students was $7.5 \pm 1.4 \%$, while the improvement for the B level and A level students was $16.8 \pm 1.4 \%$ and $15.9 \pm 1.0 \%$, respectively. 